\title{
QUANTIZATION AND PROJECTIVE REPRESENTATIONS OF SOLVABLE LIE GROUPS
}

BY

\author{
HENRI MOSCOVICI AND ANDREI VERONA
}

\begin{abstract}
ABSTRACr. Kostant's quantization procedure is applied for constructing irreducible projective representations of a solvable Lie group from symplectic homogeneous spaces on which the group acts. When specialized to a certain class of such groups, including the exponential ones, the technique exposed in the present paper provides a complete parametrization of all irreducible projective representations.
\end{abstract}

Introduction. Refining the Kirillov orbit method for the construction of the irreducible unitary representations of a nilpotent Lie group, Kostant has developed a geometric quantization theory of obtaining unitary representations for an arbitrary Lie group from symplectic manifolds on which the group acts as a transitive group of symplectic automorphisms. When applied to orbits of the coadjoint representation, which possess a canonical symplectic structure, Kostant's quantization procedure goes a long way towards constructing, in many significant cases, all (or "almost" all) the irreducible unitary representations of the given group. This method was particularly successful for solvable Lie groups, in which case it provides both a geometric criterion for being of type I and, in that case, a complete description of the unitary dual.

Besides the orbits of the coadjoint representation there are other symplectic homogeneous spaces for a Lie group $G$, for instance those which correspond to noncoboundary 2-cocycles of its Lie algebra g. In fact, when $G$ is connected and simply connected, all the simply connected symplectic homogeneous $G$-spaces arise in a canonical way from 2-cocycles in $Z^{2}(\mathrm{~g})$ (cf. [2]).

Although we cannot afford to go into details here, it must be said that, by Kostant's method, a unitary representation of $G$ can be obtained from a symplectic homogeneous space $X$ only under the additional assumption that $X$ is a Hamiltonian $G$-space $([3, \S 5])$ and this is the case if and only if $X$ covers an orbit of the coadjoint representation of $G$.

Received by the editors June 3, 1976 and, in revised form, June 22, 1977.

AMS (MOS) subject classifications (1970). Primary 22D12, 22E25.

Key words and phrases. Projective representations, solvable Lie groups, symplectic homogeneous spaces, quantization procedure. 
We have found that when the quantization procedure is applied to a general symplectic homogeneous space one can still obtain a representation of $G$, which is no longer unitary but a projective one. This remark allowed us to construct irreducible projective representations of a solvable Lie group $G$ starting from integral 2-cocycles on its Lie algebra $g$ (Theorem 5.4.1) and to classify some of them in terms of the orbits of $G$ in $Z^{2}(g)$ (Theorem 5.4.4). In the special case of a nilpotent (or, more generally, exponential) Lie group, our construction provides a complete classification of the projective dual (Corollary 5.4.5).

Now let us describe in a few words our construction of projective representations. Assume $G$ is a connected and simply connected Lie group. First, to each cocycle $\omega \in Z^{2}(g)$ we associate a strongly symplectic homogeneous $G$-space $\left(X_{\omega}, \theta_{\omega}\right)$, namely the orbit through 0 in $\mathrm{g}^{*}$ under the affine action of $G$ corresponding to $\omega$ (cf. [2]). Then, after choosing a polarization $\mathfrak{h}$ of $\mathfrak{g}$ at $\omega$, we attach to each line bundle with connection and Hermitian structure $(L, \alpha)$ over $X_{\omega}$, with curvature form $\theta_{\omega}$, a projective representation $\pi(L, \alpha ; \mathfrak{h})$ of $G$ whose equivalence class $\pi_{l, \mathfrak{h}}$ depends only on the equivalence class $l$ of $(L, \alpha)$. Under no additional hypothesis, we have nothing to say about $\pi_{l, \mathfrak{b}}$. It may be or may not be irreducible; even worse it may happen that $\pi_{l, \mathfrak{h}}=0$. However, if $G$ is assumed to be solvable, the results of Auslander and Kostant [1] allow us to conclude that $\pi_{l, \mathfrak{h}}$ is irreducible and independent of the choice of the polarization. Furthermore, $G$ acts naturally on the set of all such isomorphism classes of line bundles with connection over symplectic homogeneous $G$ spaces of the form $\left(X_{\omega}, \theta_{\omega}\right)$ with $\omega$ running through $Z^{2}(\mathrm{~g})$, and the map $l \mapsto \pi_{l}=\pi_{l, \mathfrak{h}}$ is constant on the orbits of $G$. This construction is particularly fruitful in the case of an exponential group $G$, when it yields a complete parametrization of all equivalence classes of irreducible projective representations of $G$ by the orbits of $G$ in $Z^{2}(g)$. It should be mentioned that, in principle, our method of constructing irreducible projective representations works whenever it is applied to a class of Lie groups which is closed under central extensions by $\mathbf{R}$ and for which the Kirillov-Kostant method of obtaining the irreducible unitary representations works.

The material in this paper is organized as follows. $\$ 1$ contains some known facts about extensions of Lie groups and algebras we will need later. $\$ 2$ deals with the relationship between projective representations and group extensions. $\$ 3$ is devoted to the study of the symplectic homogeneous space associated to a 2-cocycle. The concept of a polarization for a 2-cocycle is discussed in $\$ 4$. The construction of a projective representation of a Lie group $G$ by quantizing a symplectic homogeneous $G$-space is given in $\$ 5$. This section also contains the statements of the main results, while their proofs are given in the final section. 
0. Notational conventions. In order to prevent misunderstandings we list below some possibly nonstandard notations to be used in this paper.

0.1 . T stands for the group of complex numbers of modulus 1 .

0.2 . The complexification of a real vector space $V$ is denoted $V_{C}$, while the complexification of a linear (or multilinear) map $\lambda$ is denoted by the same symbol $\lambda$, without adding the subscript $\mathbf{C}$.

0.3. If a Lie group $G$ with Lie algebra $g$ acts (smoothly) on the left on a manifold $X$, we denote by $L_{X}(g)$ the diffeomorphism of $X$ defined by $g \in G$; sometimes, we shall write simply $g \cdot u$ instead of $L_{X}(g) u$, for $u \in X$.

The smooth vector field on $X$ determined by $x \in \mathfrak{g}$ is denoted by $r_{X}(x)$; recall that

$$
r_{X}(x)_{u}(f)=\left.\frac{d}{d t}(f(\exp (-t x) \cdot u))\right|_{t=0}, \quad f \in C^{\infty}(X) .
$$

When $X=G$ with the natural left $G$-action, we shall omit the subscript $G$ in the above notation. Further, we shall write $R(g)$ for the right translation by $g^{-1} \in G$ and $l(x)$ for the left invariant vector field on $G$ corresponding to $x \in \mathrm{g}$.

For $g \in G, I(g)$ denotes the inner automorphism $L(g) \circ R(g)$.

0.4. If $g$ is the Lie algebra of the Lie group $G, g^{*}$ denotes the real dual vector space of $\mathfrak{g},\langle\rangle:, \mathfrak{g}^{*} \times \mathfrak{g} \rightarrow \mathbf{R}$ the canonical pairing, $\mathrm{Ad}^{*}, \mathrm{ad}^{*}$ the coadjoint representations of $G$ and $g$ on $\mathfrak{g}^{*}$.

0.5 . If $g$ is a Lie algebra, $\iota(x)$ and $\mathscr{L}(x)$ will denote the interior product and the Lie derivative with respect to $x \in \mathfrak{g}$.

0.6. If $L$ is a smooth vector bundle over $X, \Gamma(X, L)$ stands for the vector space of all its smooth sections.

0.7. The Lie algebra of a Lie group $M$ will be sometimes denoted $\operatorname{Lie}(M)$.

\section{Extensions of Lie groups and algebras.}

1.1. Let $G$ and $K$ be two connected Lie groups. By an extension of $G$ by $K$ we shall mean an exact sequence

$$
(M, p) 1 \rightarrow K \stackrel{i}{\rightarrow} M \stackrel{p}{\rightarrow} G \rightarrow 1
$$

where $M$ is a separable locally compact group and $i, p$ are continuous homomorphisms.

Let us add some comments on this definition.

(1.1.1) $K$ being a separable locally compact group, $i: K \rightarrow \operatorname{Ker}(p)$ is in fact a homeomorphism. Owing to this remark we shall identify $K$ to $\operatorname{Ker}(p)$ via $i$, viewing $i$ as the inclusion map.

(1.1.2) Since $M$ is separable, the canonically induced map $M / K \rightarrow G$ is a homeomorphism too. This and the connectedness of $G$ and $K$ ensure that $M$ is also connected. 
(1.1.3) Moreover, $M$ admits a (unique) structure of a Lie group whose underlying topology is the original one. Indeed, to see this it suffices to note that $M$ is without small subgroups and then to apply [4, Theorem, p. 169].

When in addition $K$ is central in $M$, the extension $(M, p)$ will be called central.

The set of all central extensions of $G$ by $K$ will be denoted $\mathcal{E} x t_{0}(G, K)$. Further, we denote by $\operatorname{Ext}_{0}(G, K)$ the factor set of $\mathcal{E} t_{0}(G, K)$ with respect to the usual equivalence relation. The equivalence class of an extension $(M, p)$ will be denoted $[M, p]$.

1.2. From now on $G$ will be always assumed not only connected but also simply connected.

Let $(M, p) \in \mathcal{E} x t_{0}(G, K)$. Since $\pi_{1}(G)=0=\pi_{2}(G)$, the homotopy exact sequence of the fibration $M \stackrel{p}{\rightarrow} G$ shows that the inclusion map $K \hookrightarrow M$ induces an isomorphism between $\pi_{1}(K)$ and $\pi_{1}(M)$; therefore $M$ is simply connected if and only if $K$ is.

Now let $L$ be the simply connected covering group of $K$, with $p_{K}: L \rightarrow K$ the corresponding projection. We identify $\pi_{1}(K)$ to $\operatorname{Ker}\left(p_{K}\right)$.

Given $(M, p) \in \mathcal{E} x t_{0}(G, L)$ we shall define $\left(M_{\sharp}, p_{\sharp}\right) \in \mathcal{E} x t_{0}(G, K)$ as follows: $M_{\sharp}=M / \pi_{1}(K)$ and $p_{\sharp}: M_{\sharp} \rightarrow G$ is canonically induced by $p$ : $M \rightarrow G$. The map $(M, p) \mapsto\left(M_{\sharp}, p_{\sharp}\right)$ from $\mathcal{E} x t_{0}(G, L)$ to $\mathcal{E} x t_{0}(G, K)$ will be denoted by $\operatorname{Cov}_{\sharp}$, while the induced map from $\operatorname{Ext}_{0}(G, L)$ to $\operatorname{Ext}_{0}(G, K)$ will be denoted by $\operatorname{Cov}_{\sharp}$.

Conversely, for $(N, q) \in \mathcal{E} x t_{0}(G, K)$ let us define $\left(N^{\sharp}, q^{\sharp}\right) \in \mathcal{E} x t_{0}(G, L)$ in the following way: $N^{\#}$ is the simply connected covering group of $N$ and $q^{\sharp}=q \circ p_{N}$, where $p_{N}: N^{\sharp} \rightarrow N$ is the covering homomorphism. It is easy to check that $\operatorname{Ker}\left(q^{\sharp}\right)$ is isomorphic to $L$, hence, after identifying them, $\left(N^{\sharp}, q^{\sharp}\right)$ becomes really an extension of $G$ by $L$. The map thus defined $(N, q) \mapsto\left(N^{\sharp}, q^{\sharp}\right)$ from $\mathcal{E} x t_{0}(G, B)$ to $\mathcal{E} x t_{0}(G, L)$ is denoted by $\operatorname{Cov}^{\sharp}$ and the induced map from $\operatorname{Ext}_{0}(G, K)$ to $\operatorname{Ext}_{0}(G, L)$ is denoted $\operatorname{Cov}^{\#}$.

To conclude this subsection we note that there is no problem in verifying that $\mathrm{Cov}_{\sharp}$ and $\mathrm{Cov}^{\sharp}$ are mutually inverse maps which put in a one-to-one correspondence $\operatorname{Ext}_{0}(G, L)$ with $\operatorname{Ext}_{0}(G, K)$.

1.3. Let $\mathfrak{f}$ and $g$ denote the Lie algebras of $K$ and $G$ respectively. An extension of $\mathfrak{g}$ by $f$ is an exact sequence of Lie algebras and Lie homomorphisms

$$
(m, \psi) 0 \rightarrow \mathfrak{t} \rightarrow \mathfrak{m} \stackrel{\psi}{\rightarrow} \mathfrak{g} \rightarrow 0 .
$$

When $f$ is contained in the center of $m$, the extension will be called central.

The set of all central extensions of $g$ by $\mathfrak{f}$ is denoted $\operatorname{ext}_{0}(\mathfrak{g}, \mathfrak{f})$ and the corresponding factor set relative to the usual equivalence relation is denoted $\operatorname{ext}_{0}(\mathfrak{g}, \mathfrak{f})$. By $[\mathfrak{m}, \psi]$ we shall denote the equivalence class of the extension $(\mathfrak{m}, \psi)$. 
There is a simple relationship between $\operatorname{Ext}_{0}(G, K)$ and $\operatorname{ext}_{0}(g, f)$ which we proceed now to describe. First of all let us remark that, in view of the previous subsection, there will be no loss of generality in assuming $K$ simply connected.

Now given $(M, p) \in \mathcal{E} x t_{0}(G, K)$, by passing to Lie algebras we get a central extension $(\mathfrak{m}, \psi) \in \operatorname{ext}_{0}(\mathfrak{g}, \mathfrak{f})$ which we denote $\operatorname{Lie}^{\sharp}(M, p)$. The map $\mathcal{L i e}^{\sharp}: \mathcal{E} \mathrm{Et}_{0}(G, K) \rightarrow \operatorname{ext}_{0}(\mathfrak{g}, \mathfrak{l})$ so defined induces a map $\operatorname{Lie}^{\sharp}$ from $\operatorname{Ext}_{0}(G, K)$ to $\operatorname{ext}_{0}(\mathfrak{g}, \mathfrak{t})$.

Conversely, to each element $(\mathfrak{m}, \psi) \in \operatorname{ext}_{0}(\mathfrak{g}, \mathfrak{f})$ we associate a central extension $(M, p)=\operatorname{Lie}_{\sharp}(\mathfrak{m}, \psi)$ of $G$ by $K$, as follows: $M$ is the simply connected Lie group with Lie algebra $m$ and $K \rightarrow M, p: M \rightarrow G$ are the Lie homomorphisms whose differentials are $\mathfrak{t} \rightarrow \mathfrak{m}$ and $\psi: \mathfrak{m} \rightarrow \mathfrak{g}$, respectively. We have thus obtained a map $\mathcal{L i e}_{\sharp}: \operatorname{ext}_{0}(\mathrm{~g}, \mathfrak{t}) \rightarrow \mathcal{E} x t_{0}(G, K)$, which induces a map $\operatorname{Lie}_{\sharp}$ from $\operatorname{ext}_{0}(\mathfrak{g}, \mathfrak{f})$ to $\operatorname{Ext}_{0}(G, K)$.

It is only a trivial observation to remark that $\mathrm{Lie}_{\#}$ and Lie ${ }^{\sharp}$ are mutually inverse maps between $\operatorname{ext}_{0}(\mathfrak{g}, \mathfrak{l})$ and $\operatorname{Ext}_{0}(G, K)$.

1.4. By $Z^{2}(g)$ we shall denote, as usual, the vector space of all 2-cocycles on $g$ relative to the trivial action of $g$ on $R$.

For each $\omega \in Z^{2}(g)$ one defines a central extension $\left(m_{\omega}, \psi_{\omega}\right)$ of $g$ by $\mathbf{R}$ in the following way: $\mathfrak{m}_{\omega}$ is the Lie algebra whose underlying vector space is $\mathbf{R} \times \mathfrak{g}$, the bracket operation being given by the formula

$$
[(r, x),(s, y)]=(-\omega(x, y),[x, y]), \quad r, s \in \mathbf{R}, x, y \in g ;
$$

the projection $\psi_{\omega}: \mathfrak{m} \rightarrow g$ is just the canonical projection of $\mathbf{R} \times g$ onto $g$, and finally $\mathbf{R} \rightarrow \mathfrak{m}_{\omega}$ is the canonical injection of $\mathbf{R}$ into $\mathbf{R} \times \mathfrak{g}$.

Now if $\omega^{\prime}=\omega+d \lambda$, with $\lambda \in \mathrm{g}^{*}$, then the Lie homomorphism $\varphi_{\lambda}: \mathrm{m}_{\omega} \rightarrow$ $\mathrm{m}_{\omega^{\prime}}$ given by

$$
\varphi_{\lambda}(r, x)=(r+\lambda(x), x), \quad r \in \mathbf{R}, x \in \mathfrak{g},
$$

establishes an equivalence of extensions between $\left(\mathfrak{m}_{\omega}, \psi_{\omega}\right)$ and $\left(\mathfrak{m}_{\omega^{\prime}}, \psi_{\omega^{\prime}}\right)$. It follows that the assignment $\omega \in Z^{2}(g) \mapsto\left[\mathfrak{m}_{\omega}, \psi_{\omega}\right] \in \operatorname{ext}_{0}(\mathfrak{g}, \mathbf{R})$ gives rise to a map $[\omega] \mapsto\left[\mathfrak{m}_{\omega}, \psi_{\omega}\right]$ from $H^{2}(g)$ to $\operatorname{ext}_{0}(g, \mathbf{R})$. It is well known that this map is in fact a bijection.

2. Projective representations and group extensions. Given a separable Hilbert space $\mathbf{H}$ we denote by $\mathbf{U}(\mathbf{H})$ the group of all its unitary automorphisms, endowed with the strong operatorial topology. Further we denote by $\mathbf{P U}(\mathbf{H})$ the projective unitary group $\mathbf{U}(\mathbf{H}) / \mathbf{T}$, where the circle group $T$ is viewed as the normal closed subgroup of $\mathbf{U}(\mathbf{H})$ consisting of scalar multiples of the identity operator Id, and we give PU(H) the quotient of the strong topology; $p_{\mathbf{H}}: \mathbf{U}(\mathbf{H}) \rightarrow \mathbf{P U}(\mathbf{H})$ stands for the canonical projection. It is convenient to regard an element of $\mathbf{P U}(\mathbf{H})$ as an automorphism of the projective space $\mathbf{P H}$ associated to $\mathbf{H}$. 
By a unitary (resp. projective) representation of $G$ in $\mathbf{H}$ we mean a continuous homomorphism of $G$ in $\mathbf{U}(\mathbf{H})$ (resp. PU(H)). Recall that two projective representations $\pi_{i}: G \rightarrow \mathbf{P U}\left(\mathbf{H}_{i}\right), i=1,2$, are said to be projectively equivalent if there exists a unitary isomorphism $U: \mathbf{H}_{1} \rightarrow \mathbf{H}_{2}$ such that, if $\hat{U}$ : $\mathbf{P H}_{1} \rightarrow \mathbf{P H}_{2}$ denotes the corresponding isomorphism of projective spaces, then $\hat{U} \circ \pi_{1}(g)=\pi_{2}(g) \circ \hat{U}$. The set of all equivalence classes of irreducible projective representations of $G$, which we call the projective dual of $G$, will be denoted $G^{\mathrm{n}}$.

2.1. Let $(M, p)$ be a central extension of the connected and simply-connected Lie group $G$ by $R$. A unitary representation $\rho: M \rightarrow U(H)$ will be called projectable if $\rho(r)=\exp \{2 \pi i r\}$ Id, $r \in \mathbf{R}$. In this case there exists a unique projective representation $\hat{\rho}: G \rightarrow \mathbf{P U}(\mathbf{H})$ such that the diagram

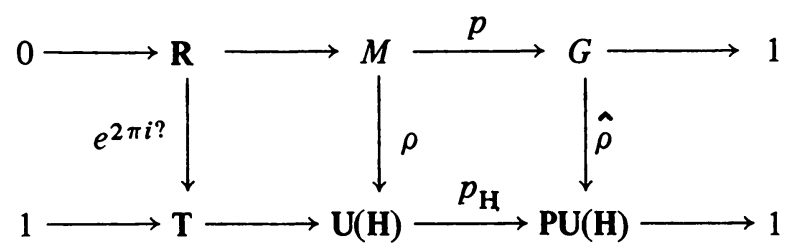

commutes.

Now let $\rho_{i}: M \rightarrow \mathbf{U}\left(\mathbf{H}_{i}\right), i=1,2$, be projectable unitary representations such that $\hat{\rho}_{1}$ and $\hat{\rho}_{2}$ are projectively equivalent through the unitary isomorphism $U: \mathbf{H}_{1} \rightarrow \mathbf{H}_{2}$. For each $m \in M$ one has

$$
p_{\mathbf{H}}\left(\rho_{2}(m)\right)=\hat{\rho}_{2}(p(m))=\hat{U} \circ \rho_{1}(p(m)) \circ \hat{U}^{-1}=p_{\mathbf{H}}\left(U \circ \rho_{1}(m) \circ U^{-1}\right) ;
$$

hence there exists a unitary character $\chi: M \rightarrow T$ such that

$$
\rho_{2}(m)=\chi(m) \cdot U \circ \rho_{1}(m) \circ U^{-1}, \quad m \in M .
$$

This means that $\rho_{2}$ and $\chi \otimes \rho_{1}$ are unitarily equivalent representations of $M$.

2.2. We want now to attach to a given projective representation $\pi: G \rightarrow$ PU(H) an extension $\left(M_{\pi}, p_{\pi}\right) \in \mathcal{E} x t_{0}(G, R)$ together with a unitary representation $\check{\pi}: \mathbf{M} \rightarrow \mathbf{U}(\mathbf{H})$, and then to relate this construction to that discussed in 2.1.

Consider the topological subgroup $N_{\pi}$ of $\mathrm{U}(\mathrm{H}) \times G$ consisting of those pairs $(u, g)$ which satisfy $p_{\mathbf{H}}(u)=\pi(g)$. Define $\mathbf{T} \rightarrow N_{\pi}$ to be $t \mapsto(t \cdot \mathrm{Id}, 1)$ and $q_{\pi}: N_{\pi} \rightarrow G$ by $q_{\pi}(u, g)=g$. Clearly

$$
\left(N_{\pi}, q_{\pi}\right) 1 \rightarrow \mathbf{T} \rightarrow N_{\pi} \stackrel{q_{\pi}}{\rightarrow} G \rightarrow 1
$$

is an exact sequence of topological groups and continuous homomorphisms. Moreover, $q_{\pi}$ is an open map (since $p_{\mathbf{H}}$ is so) hence $N_{\pi} / \mathbf{T}$ is homeomorphic to $G$. It follows that $N_{\pi}$ is a (separable) locally compact group. Therefore, $\left(N_{\pi}, q_{\pi}\right) \in \mathcal{E x t}_{0}(G, \mathbf{T})$.

Let us now define $\left(M_{\pi}, p_{\pi}\right) \in \mathcal{E} x t_{0}(G, \mathbf{R})$ to be $\operatorname{Cov}^{\sharp}\left(N_{\pi}, q_{\pi}\right)$. The map 
$(u, g) \mapsto u$ is a unitary representation of $N_{\pi}$, which when composed with the covering homomorphism $M_{\pi} \rightarrow N_{\pi}$ gives rise to a unitary representation $\check{\pi}$ : $M_{\pi} \rightarrow \mathbf{U}(\mathbf{H})$. It is an easy matter to see that $\rho=\check{\pi}$ is projectable and that $\hat{\rho}=\pi$.

Conversely, let $(M, p) \in \mathcal{E} x t_{0}(G, \mathbf{R})$ and $\rho: M \rightarrow \mathbf{U}(\mathbf{H})$ be a projectable unitary representation. Put $\pi=\hat{\rho}$ and consider, as above, the associated extensions $\left(N_{\pi}, q_{\pi}\right) \in \mathcal{E}_{x t_{0}}(G, \mathbf{T})$ and $\left(M_{\pi}, p_{\pi}\right) \in \mathcal{E x t}_{0}(G, \mathbf{R})$. Define now $\Psi$ : $M \rightarrow N_{\pi}$ by $\Psi(m)=(\rho(m), p(m)), m \in M$, and then form the diagram

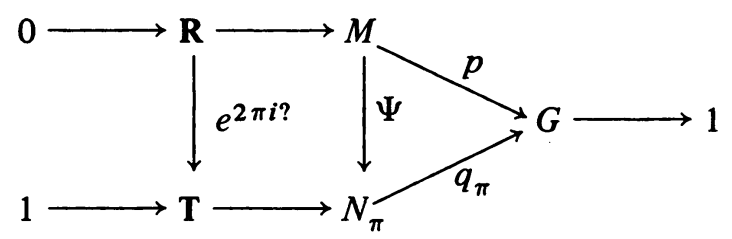

which, obviously, commutes. Since $M$ is simply connected $\Psi$ can be lifted to a Lie homomorphism $\Phi: M \rightarrow M_{\pi}$ which makes the following diagram commutative:

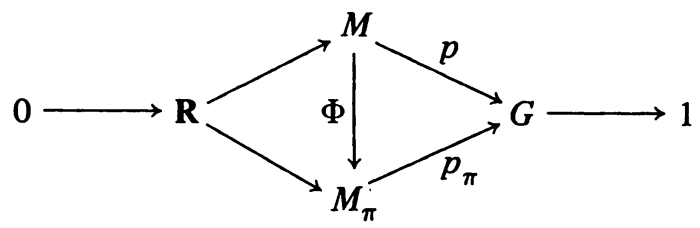

Actually, $\Phi$ is an equivalence between the extensions $(M, p)$ and $\left(M_{\pi}, p_{\pi}\right)$. In addition one has $\rho=\check{\pi} \circ \Phi$.

2.3. Let $\pi_{i}: G \rightarrow \mathbf{P U}\left(\mathbf{H}_{i}\right), i=1,2$, be projectively equivalent representations, via the unitary isomorphism $U: \mathbf{H}_{1} \rightarrow \mathbf{H}_{2}$. It is not difficult to see that the isomorphism $\Psi_{U}: N_{\pi_{1}} \rightarrow N_{\pi_{2}}$, defined by

$$
\Psi_{U}(u, g)=\left(U \circ u \circ U^{-1}, g\right), \quad(u, g) \in N_{\pi_{1}},
$$

establishes an equivalence of extensions between $\left(N_{\pi_{1}}, q_{\pi_{1}}\right)$ and $\left(N_{\pi_{2}}, q_{\pi_{2}}\right)$. Then $\Phi_{U}: M_{\pi_{1}} \rightarrow M_{\pi_{2}}$, the lifting of $\Psi_{U}$ to the simply connected covering groups, establishes an equivalence of extensions between $\left(M_{\pi_{1}}, p_{\pi_{1}}\right)$ and $\left(M_{\pi_{2}}, p_{\pi_{2}}\right)$. Furthermore, $\check{\pi}_{1}$ and $\check{\pi}_{2} \circ \Phi_{U}$ are unitarily equivalent representations.

2.4. Suppose now that $\left(M_{i}, p_{i}\right) \in \mathcal{E} x t_{0}(G, \mathbf{R}), i=1,2$, and that $\rho_{i}: M_{i} \rightarrow$ $\mathbf{U}\left(\mathbf{H}_{i}\right), i=1,2$, are projectable unitary representations whose associated projective representations $\hat{\rho}_{i}: G \rightarrow \mathbf{P U}\left(\mathbf{H}_{i}\right)$ are projectively equivalent. Then, by combining 2.2 and 2.3 , one can see without difficulty that there exists a Lie isomorphism $\Phi: M_{1} \rightarrow M_{2}$ which determines an equivalence of extensions 
between $\left(M_{1}, p_{1}\right)$ and $\left(M_{2}, p_{2}\right)$ and has the further property that $\rho_{1}$ and $\rho_{2} \circ \Phi$ are unitarily equivalent representations.

3. The symplectic homogeneous space associated to a 2-cocycle. In this section $\omega$ will denote a fixed element in $Z^{2}(g), g$ being, as we have already agreed, the Lie algebra of the connected and simply connected Lie group $G$.

3.1. To begin with, we shall define a representation $\tau_{\omega}$ of $g$ on $\mathbf{R} \times g^{*}$ by the formula

$$
\tau_{\omega}(x)(r, \lambda)=\left(0, \operatorname{ad}^{*}(x) \lambda+r \iota(x) \omega\right), \quad x \in \mathfrak{g},(r, \lambda) \in \mathbf{R} \times g^{*} .
$$

The group $G$ being simply connected, there exists a unique representation $T_{\omega}$ of $G$ on $\mathbf{R} \times \mathrm{g}^{*}$ whose differential is $\tau_{\omega}$. An easy computation shows that

$$
T_{\omega}(g)(0, \lambda)=\left(0, \operatorname{Ad}^{*}(g) \lambda\right), \quad g \in G, \lambda \in g^{*} .
$$

On the other hand we observe that $T_{\omega}$ must verify a relation of the form

$$
T_{\omega}(g)(r, 0)=\left(r, r F_{\omega}(g)\right), \quad g \in G, r \in \mathbf{R},
$$

with $F_{\omega}: G \rightarrow g^{*}$ analytic. Since $T_{\omega}$ is a representation, one can see that

$$
F_{\omega}(g h)=\operatorname{Ad}^{*}(g) F_{\omega}(h)+F_{\omega}(g), \quad g, h \in G .
$$

Now taking into account the fact that the differential of $T_{\omega}$ is $\tau_{\omega}$, one deduces that $F_{\omega}$ has the expression

$$
F_{\omega}(\exp x)=\sum_{n=1}^{\infty} \frac{1}{n !}\left(\operatorname{ad}^{*}(x)\right)^{n-1}(\iota(x) \omega), \quad x \in \mathfrak{g} .
$$

Finally, one obtains

$$
T_{\omega}(g)(r, \lambda)=\left(r, \operatorname{Ad}^{*}(g) \lambda+r F_{\omega}(g)\right), \quad g \in G,(r, \lambda) \in \mathbf{R} \times g^{*} .
$$

Let us denote by $\mathrm{Ad}^{*}$ too the natural representation of $G$ on $Z^{2}(\mathrm{~g})$. Explicitly, $\left(\operatorname{Ad}^{*}(g) \sigma\right)(x, y)=\sigma\left(\operatorname{Ad}\left(g^{-1}\right) x, \operatorname{Ad}\left(g^{-1}\right) y\right)$, for $g \in G, \quad \sigma \in$ $Z^{2}(\mathrm{~g}), x, y \in \mathfrak{g}$.

Starting from (3.1.2) one can easily see that

$$
F_{\mathrm{Ad}^{*}(g) \omega}(\exp x)=\operatorname{Ad}^{*}(g) F_{\omega}\left(g^{-1} \cdot \exp x \cdot g\right), \quad g \in G, x \in g,
$$

which, when combined with (3.1.1), leads to the formula

$$
F_{\mathrm{Ad}^{*}(g) \omega}(h)=\operatorname{Ad}^{*}(g) F_{\omega}\left(g^{-1} h g\right), \quad g, h \in G .
$$

Consider now the linear map $D_{\omega}: \mathbf{R} \times g^{*} \rightarrow Z^{2}(g)$, given by $D_{\omega}(r, \lambda)=d \lambda$ $+r \omega$. One has

$$
D_{\omega} \circ \tau_{\omega}(x)=\mathcal{L}(x) \circ D_{\omega}, \quad x \in \mathfrak{g} .
$$

As $x \mapsto \mathfrak{L}(x)$ from $g$ to $g l\left(Z^{2}(g)\right)$ is the differential of the homomorphism $\mathrm{Ad}^{*}$ : $G \rightarrow G L\left(Z^{2}(g)\right)$, it follows that

$$
D_{\omega} \circ T_{\omega}(g)=\operatorname{Ad}^{*}(g) \circ D_{\omega}, \quad g \in G,
$$


which is equivalent to

$$
\operatorname{Ad}^{*}(g) \omega-\omega=d\left(F_{\omega}(g)\right), \quad g \in G .
$$

3.2. We maintain the above notation. In addition, let us denote: $G(\omega)=\{g$ $\left.\in G ; \quad F_{\omega}(g)=0\right\}$ and $g(\omega)=\{x \in g ; \quad l(x) \omega=0\}$. The formula (3.1.1) implies that $G(\omega)$ is a (closed) subgroup of $G$ and that $G(\omega)=\{g \in G$; $F_{\omega}(h g)=F_{\omega}(h)$ for any $\left.h \in G\right\}$. Therefore, $F_{\omega}$ factorizes through a map $f_{\omega}$ : $G / G(\omega) \rightarrow g^{*}$.

Since Lie $G(\omega)=\{x \in \mathfrak{g} ; \exp t x \in G(\omega)$ for any $t \in \mathbf{R}\}$ and, on the other hand, $\exp t x \in G(\omega)$ for any $t \in \mathbf{R}$ if and only if $\iota(x) \omega=0$ (cf. (3.1.2)), one gets

$$
\text { Lie } G(\omega)=g(\omega) .
$$

Furthermore, we observe that, in view of (3.1.6), one has

$$
\operatorname{Ad}^{*}(g) \omega=\omega \text { for any } g \in G(\omega) \text {. }
$$

Now set $X_{\omega}=G / G(\omega)$ and let $q_{\omega}: G \rightarrow X_{\omega}$ be the canonical projection. From the definition of $g(\omega),(3.2 .1)$ and (3.2.2), we infer that there exists a unique $G$-invariant closed 2-form $\theta_{\omega}$ on $X_{\omega}$ such that $\left(q_{\omega}^{*}\left(\theta_{\omega}\right)\right)_{1}=\omega$, where $1 \in G$ is the unit element.

To simplify the notation, when no confusion can arise, we shall write shortly $X, q, \theta, F, f$ instead of $X_{\omega}, q_{\omega}, \theta_{\omega}, F_{\omega}, f_{\omega}$, respectively.

For each $x \in \mathfrak{g}$ let us define $f^{x} \in C^{\infty}(X)$ by

$$
f^{x}(q(g))=\langle F(g), x\rangle, \quad g \in G .
$$

It is an easy matter to see that the vector field $r_{X}(x)$, defined as in 0.3 , satisfies the following relations:

$$
\begin{gathered}
L_{X}(g)_{*} r_{X}(x)_{u}=r_{X}(\operatorname{Ad}(g) x)_{g \cdot u}, \quad g \in G, u \in X \\
r_{X}(x)_{q(g)}=-q_{*}\left(L(g)_{*}\left(\operatorname{Ad}\left(g^{-1}\right) x\right)\right), \quad g \in G .
\end{gathered}
$$

3.2.1. Proposition. $\left(X_{\omega}, \theta_{\omega}\right)$ is a strongly symplectic $G$-space.

Proof. Precisely, we shall prove that

$$
\iota\left(r_{X}(x)\right) \theta=d f^{x} \quad \text { for any } x \in g .
$$

To this end, let us note first that, since $q^{*}$ is injective, it suffices to check that

$$
q^{*}\left(\iota\left(r_{X}(x)\right) \theta\right)=q^{*}\left(d f^{x}\right), \quad x \in \mathfrak{g} .
$$


Now let $g \in G$ and $x \in \mathfrak{g}$. One has, for any $y \in \mathfrak{g}$,

$$
\begin{aligned}
\left\langle\left(q^{*}\left(\iota\left(r_{X}(x)\right) \theta\right)\right)_{g}, L(g)_{*} y\right\rangle & =\left\langle\left(\iota\left(r_{X}(x)\right) \theta\right)_{q(g)}, L_{X}(g)_{*} \circ q_{*}(y)\right\rangle \\
& =\theta_{q(g)}\left(r_{X}(x)_{q(g)}, L_{X}(g)_{*} \circ q_{*}(y)\right) \\
& =\left(L_{X}\left(g^{-1}\right)^{*} \theta_{q(1)}\right)\left(r_{X}(x)_{q(g)}, L_{X}(g)_{*} \circ q_{*}(y)\right) \\
& =\theta_{q(1)}\left(L_{X}\left(g^{-1}\right)_{*} r_{X}(x)_{q(g)}, q_{*}(y)\right) \\
& =\theta_{q(1)}\left(-q_{*}\left(\operatorname{Ad}\left(g^{-1}\right) x\right), q_{*}(y)\right) \\
& =\omega\left(y, \operatorname{Ad}\left(g^{-1}\right) x\right) .
\end{aligned}
$$

On the other hand,

$$
\begin{aligned}
\left\langle\left(q^{*}\left(d f^{x}\right)\right)_{g}, L(g)_{*} y\right\rangle & =\left\langle d\left(f^{x} \circ q\right)_{g}, L(g)_{*} y\right\rangle=\left.\frac{d}{d t}\langle F(g \exp t y), x\rangle\right|_{t=0} \\
& =\left.\frac{d}{d t}\left\langle\operatorname{Ad}^{*}(g) F(\exp t y), x\right\rangle\right|_{t=0}+\left.\frac{d}{d t}\langle F(g), x\rangle\right|_{t=0} \\
& =\left\langle\operatorname{Ad}^{*}(g)(\iota(y) \omega), x\right\rangle=\omega\left(y, \operatorname{Ad}^{*}\left(g^{-1}\right) x\right) .
\end{aligned}
$$

This proves our assertion.

3.2.2. REMARK. In contrast with the construction given in [3] for the case when $\omega$ is a coboundary, the map $x \mapsto f^{x}$ is no more a Lie homomorphism with respect to the Poisson bracket on $C^{\infty}(X)$. However, it satisfies

$$
\left[f^{x}, f^{y}\right]=f^{[x, y]}-\omega(x, y), \quad x, y \in \mathfrak{g} .
$$

3.3. We shall say that $\omega$ is an integral 2-cocycle if the cohomology class $\left[\theta_{\omega}\right] \in H^{2}\left(X_{\omega}, \mathbf{R}\right)$ is integral. This integrality property is preserved by the action of $G$ on $Z^{2}(g)$. Indeed, if $\omega^{\prime}=\operatorname{Ad}^{*}(g) \omega$ with $g \in G$, then from (3.1.4) it follows that $G\left(\omega^{\prime}\right)=I(g) G(\omega)$, hence $I(g)$ induces a diffeomorphism $i(g)$ : $X_{\omega} \rightarrow X_{\omega^{\prime}}$ such that $i(g)^{*} \theta_{\omega^{\prime}}=\theta_{\omega}$. This means that $i(g)$ is an isomorphism of symplectic spaces. In particular, it preserves the integrality condition.

The subspace of all integral 2-cocycles in $Z^{2}(\mathrm{~g})$ will be denoted $Z_{0}^{2}(\mathrm{~g})$.

\section{Polarizations.}

4.1. A complex subalgebra $\mathfrak{h} \subset g_{c}$ will be called a polarization of $\mathfrak{g}$ at $\omega \in Z^{2}(g)$ if it satisfies:

(i) $\mathfrak{h}$ is a maximally isotropic subspace of $g_{\mathbf{c}}$ relative to $\omega$;

(ii) $\mathfrak{h}+\overline{\mathfrak{h}}$ is a Lie subalgebra of $\mathfrak{g}_{\mathfrak{c}}$;

(iii) $\mathfrak{h}$ is $\operatorname{Ad}_{G} G(\omega)$-stable.

If, in addition, $\mathfrak{h}$ fulfils the condition

(iv) $i \omega(x, \bar{x}) \leqslant 0$ for any $x \in \mathfrak{h}$, it is called a positive polarization.

By $\mathfrak{n}$ we denote the nil-radical of $g$. Set $G(\omega \mid \mathfrak{n})=\left\{g \in G ; F_{\omega}(g) \mid \mathfrak{n}=0\right\}$. 
Now we shall say that the polarization $\mathfrak{h}$ is nil-admissible if it has the property:

(v) $\mathfrak{h} \cap \mathfrak{n}_{\mathbf{C}}$ is a maximally isotropic subspace of $\mathfrak{n}_{\mathbf{C}}$ relative to $\omega \mid \mathfrak{n}_{\mathbf{C}} \times \mathfrak{n}_{\mathbf{C}}$ and it is $\operatorname{Ad}_{G} G(\omega \mid \mathfrak{n})$-stable.

From now on $\mathfrak{h}$ will be a fixed polarization of $\mathfrak{g}$ at $\omega$. Consider the Lie subalgebras of $\mathfrak{g}: \mathfrak{d}=\mathfrak{h} \cap \mathfrak{g}$ and $e=(\mathfrak{h}+\overline{\mathfrak{h}}) \cap \mathfrak{g}$. Then it is easily seen that:

$$
\begin{gathered}
\mathfrak{g}(\omega) \subset \mathfrak{d} \subset \mathrm{e} ; \\
\mathfrak{d}_{\mathbf{C}}=\mathfrak{h} \cap \overline{\mathfrak{h}} \text { and } e_{\mathbf{C}}=\mathfrak{h}+\overline{\mathfrak{h}} ;
\end{gathered}
$$

(4.1.3) $\delta$ is the orthogonal subspace of $e$ relative to $\omega$ and thus the canonically induced 2 -form $\hat{\omega}$ on $e / \delta$ is nondegenerate.

Let $D_{0}$ and $E_{0}$ denote the connected Lie subgroups of $G$ which correspond to the subalgebras $\delta$ and e respectively. Since $\mathfrak{h}$ is stable under $\operatorname{Ad}_{G} G(\omega), D_{0}$ and $E_{0}$ are normalized by $G(\omega)$. It follows that $D=D_{0} G(\omega)$ and $E=$ $E_{0} G(\omega)$ are subgroups of $G$.

With this notation, we shall say that $\mathfrak{h}$ is a closed polarization if $F_{\omega}(E)$ $\left(=F_{\omega}\left(E_{0}\right)\right)$ is a closed set in $g^{*}$.

4.2. The notation being as in 1.4 , let $\left(\mathfrak{m}_{\omega}, \psi_{\omega}\right) \in \operatorname{ext}_{0}(\mathrm{~g}, \mathbf{R})$ and $\left(M_{\omega}, p_{\omega}\right)=$ $\operatorname{Lie}_{\sharp}\left(\mathfrak{m}_{\omega}, \psi_{\omega}\right) \in \mathcal{E} x t_{0}(G, \mathbf{R})$ be the extensions associated to the fixed 2-cocycle $\omega$. We shall identify $\mathfrak{m}_{\omega}^{*}$ to $\mathbf{R} \times \mathfrak{g}^{*}$ in the obvious manner. Since $\mathbf{R} \subset \mathfrak{m}_{\omega}$ is central, the coadjoint representation of $\mathfrak{m}_{\omega}$ factorizes through a representation of $g$ in $m_{\omega}^{*}=\mathbf{R} \times g^{*}$, which is just $\tau_{\omega}$. It follows that one has

$$
T_{\omega}\left(p_{\omega}(m)\right)=\operatorname{Ad}^{*}(m) \text { for any } m \in M_{\omega} \text {. }
$$

Define $\nu_{\omega} \in \mathfrak{m}_{\omega}^{*}$ as being $(1,0) \in \mathbf{R} \times g^{*}$. Since there is no danger of confusion, we shall drop in the sequel the subscript $\omega$ in $\mathrm{m}_{\omega}, \psi_{\omega}, \nu_{\omega}, M_{\omega}, p_{\omega}$.

We will now list some facts which can be verified in a straightforward way:

$$
\psi^{*}(\omega)=d \nu
$$

(4.2.3) $\mathfrak{h} \subset g_{\mathbf{C}}$ is a polarization of $\mathfrak{g}$ at $\omega$ if and only if $\tilde{\mathfrak{h}}=\psi^{-1}(\mathfrak{h}) \subset \mathfrak{m}_{\mathbf{C}}$ is a polarization of $\mathfrak{m}$ at $\nu$ (in the sense of [1]);

(4.2.4) $\mathfrak{h}$ is a positive polarization at $\omega$ if and only if $\tilde{\mathfrak{h}}$ is a positive polarization at $\nu$;

(4.2.5) let $D, E$ be the groups associated to the polarization $\mathfrak{h}$ as above, and let $\tilde{D}, \tilde{E}$ denote the groups associated to $\tilde{\mathfrak{h}}$ as in $[1, I .5]$; then $\tilde{D}=p^{-1}(D)$ and $\tilde{E}=p^{-1}(E)$;

(4.2.6) the polarization $\mathfrak{h}$ is closed (resp. nil-admissible) if and only if $\tilde{\mathfrak{h}}$ satisfies the Pukanszky condition (resp. is strongly admissible).

These remarks together with Proposition I.5.1 and Proposition I.5.4 in [1] give the following consequences:

(4.2.7) with the above notation, $D$ is closed in $G$ and $D_{0}$ is its identity component; 
(4.2.8) if $\mathfrak{h}$ is a closed polarization, then $E$ is closed in $G$ and $E_{0}$ is the identity component of $E$.

Furthermore, one has

(4.2.9) if $\mathfrak{h}$ is a closed polarization at $\omega$, then $F_{\omega}(D)$ is a linear variety in $\mathfrak{g}^{*}$.

Indeed, from (4.2.1) and (3.1.3) we deduce that $\left\{\operatorname{Ad}_{M}(\tilde{d}) \nu ; \tilde{d} \in \tilde{D}\right\}=$ $\left\{\left(1, F_{\omega}(d)\right) ; d \in D\right\} \subset \mathbf{R} \times \mathrm{g}^{*}$, and our last claim is now a direct consequence of Proposition I.5.6 in [1].

Finally, let us observe that, in view of (4.2.6) and Proposition II.2.5 in [1], one has

(4.2.10) if $G$ is solvable, any nil-admissible polarization of $g$ at $\omega$ is closed.

4.3. From now on we shall suppose that $\mathfrak{h}$ is a positive, closed polarization of $g$ at $\omega$. Since the 2 -form $\omega \mid e \times e$ is $\operatorname{Ad}_{E}^{*} D$-invariant and $\iota(y)(\omega \mid e \times e)=0$ for any $y \in \delta, \omega$ induces an $E$-invariant 2-form on $E / D$ which, by (4.1.3), is nondegenerate. In particular, there exists on $E / D$ an $E$-invariant volume element. Therefore, the modular function $\Delta_{D}$ of $D$ coincides with the restriction to $D$ of the modular function $\Delta_{E}$ of $E$. We pick now a function $\beta \in C^{\infty}(G)$, strictly positive and such that $\beta(1)=1$ and $\beta(g e)=$ $\Delta_{E}(e) \Delta_{G}(e)^{-1} \beta(g)$ for any $g \in G, e \in E$. Then, for each $g \in G$ we define the function $\beta_{g} \in C^{\infty}(G / D)$ by $\beta_{g}\left(q_{D}(a)\right)=\beta\left(g^{-1} a\right) \beta(a)^{-1}, a \in G$, where $q_{D}$ : $G \rightarrow G / D$ is the canonical projection.

\subsubsection{Lemma. If $x \in \mathfrak{h}, g, a \in G$, then $\left(q_{D}\right)_{*}\left(L(a)_{*} x\right) \beta_{g}=0$.}

Proof. Actually, the stated formula is valid even for $x \in e$. Indeed, one has

$$
\begin{aligned}
\left(q_{D}\right)_{*}\left(L(a)_{*} x\right) \beta_{g} & =\left.\frac{d}{d t}\left(\beta_{g}\left(q_{D}(a \cdot \exp t x)\right)\right)\right|_{t=0} \\
& =\left.\frac{d}{d t}\left(\frac{\beta\left(g^{-1} a \cdot \exp t x\right)}{\beta(a \cdot \exp t x)}\right)\right|_{t=0}=\left.\frac{d}{d t}\left(\frac{\beta\left(g^{-1} a\right)}{\beta(a)}\right)\right|_{t=0}=0 .
\end{aligned}
$$

Before finishing this subsection, let us fix one more notation: $\mu$ will stand for the quasi-invariant measure on $G / D$ which corresponds to $\beta$ and to a choice of a left Haar measure on $G$. Recall that, for any $f \in C_{c}(G / D)$ and $g \in G$, one has

$$
\int_{G / D} \beta_{g}(u) f\left(g^{-1} u\right) d \mu(u)=\int_{G / D} f(u) d \mu(u) .
$$

5. Projective representations constructed by the quantization procedure. Throughout this section $\omega$ will be an integral cocycle in $Z^{2}(\mathfrak{g}), \mathfrak{h}$ will denote a closed, positive polarization of $g$ at $\omega, D, E$ will be the associated closed subgroups of $G ; X=X_{\omega}, q=q_{\omega}, \theta=\theta_{\omega}, F=F_{\omega}$ have the same meaning as in 3.2.

5.1. As in [3], let $\mathscr{L}_{c}(X, \theta)$ denote the set of all equivalence classes of 
complex line bundles with connection and invariant Hermitian structure over $X$, with curvature form $\theta$. Since $\omega$ is assumed to be integral, $\mathfrak{L}_{c}(X, \theta)$ is nonvoid (cf. [3, Proposition 2.1.1]). Pick $\ell \in \mathcal{L}_{c}(X, \theta),(L, \alpha) \in \ell$ and let $p r_{L}$ : $L \rightarrow X$ denote the corresponding projection. Now let $\mathscr{D}_{\ell}(X)$ be the group of diffeomorphisms of $X$ which leave $\ell$ unchanged, and let $\mathcal{E}(L, \alpha)$ be the group of all diffeomorphisms of $L$ which commute with the scalar multiplication and preserve both the connection form and the Hermitian structure. Then, according to Theorem 1.13.1 in [3], one has an exact sequence of groups

$$
1 \rightarrow \mathbf{T} \rightarrow \mathcal{E}(L, \alpha) \rightarrow \mathscr{D}_{\ell}(X) \rightarrow 1,
$$

where the injection $\mathbf{T} \rightarrow \mathcal{E}(L, \alpha)$ is defined by the scalar action of $\mathbf{T}$ on $L$, and the projection $\mathcal{E}(L, \alpha) \rightarrow \mathscr{D}_{\ell}(X)$ is given by $e \mapsto \hat{e}, \hat{e}$ denoting the unique diffeomorphism of $X$ such that $p r_{L} \circ e=\hat{e} \circ p r_{L}$.

Consider now the projection $p_{D}: X=G / G(\omega) \rightarrow G / D$ with fibre $D / G(\omega)$. Since $F$ induces a diffeomorphism of $D / G(\omega)$ onto $F(D),(4.2 .9)$ implies that $D / G(\omega)$ is connected and simply connected. Note also that $\theta$ vanishes on the fibres of $p_{D}$. These two remarks ensure that the parallel transport along the curves which are completely contained in the fibres of $p_{D}$ depends only on their extremities. Thus, for any two points $u, v \in X$ such that $p_{D}(u)=p_{D}(v)$, one can define unambiguously an isometry $P_{u, v}: L_{u} \rightarrow L_{v}$, namely that given by the parallel transport along any curve contained in $p_{D}^{-1}\left(p_{D}(u)\right)$ with initial point $u$ and end point $v$.

Define now an equivalence relation on $L$ as follows: $a \sim b$ if $p_{D}(u)=$ $p_{D}(v)$ and $P_{u, v}(a)=b$, where $u=p r_{L}(a)$ and $v=p r_{L}(b)$. The corresponding quotient space will be denoted $L / D$, while $\tilde{p}_{D}: L \rightarrow L / D$ will stand for the canonical projection. It is perfectly clear that $p r_{L}$ factorizes through a map $p r_{L / D}: L / D \rightarrow G / D$ such that the diagram

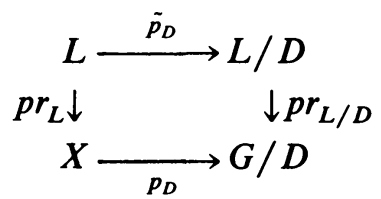

commutes. Moreover, $p r_{L / D}: L / D \rightarrow G / D$ is a complex line bundle with Hermitian structure $\langle$,$\rangle inherited from the Hermitian structure of L$.

5.2. Let all notation be as above. Consider the vector space $\Gamma_{D}(X, L, \alpha)$ consisting of all sections $s \in \Gamma(X, L)$ such that $\nabla_{\xi} s=0$ for any tangent vector $\xi$ with $\left(p_{D}\right)_{*} \xi=0$. It is an easy matter to see that for any two points $u$, $v \in X$ with $p_{D}(u)=p_{D}(v)$ one has $P_{u, v}(s(u))=s(v)$; hence $s$ determines a section $s_{D} \in \Gamma(G / D, L / D)$.

5.2.1. LEMMA. The map $s \mapsto s_{D}$ establishes a one-to-one correspondence between $\Gamma_{D}(X, L, \alpha)$ and $\Gamma(G / D, L / D)$. 
The proof is straightforward, so we will omit it.

5.3. Consider now the subspace $\mathcal{H}(L, \alpha ; \mathfrak{h})$ of $\Gamma_{D}(X, L, \alpha)$ consisting of those sections $s \in \Gamma(X, L)$ which satisfy:

$$
\begin{gathered}
\nabla_{q_{*}\left(L(g)_{*} x\right)} s=0 \text { for any } x \in \mathfrak{h} \text { and } g \in G ; \\
\int_{G / D}\left\|s_{D}(u)\right\|^{2} d \mu(u)<\infty .
\end{gathered}
$$

Take then the obvious scalar product on $\mathcal{H}(L, \alpha ; \mathfrak{h})$ and let $\mathbf{H}(L, \alpha ; \mathfrak{h})$ be the associated Hilbert space.

We intend to define a projective representation of $G$ on $\mathbf{H}(L, \alpha ; \mathfrak{h})$. To this end let us consider $g \in G$ and note that, in view of Remark 4.4.2 in [3] and our Proposition 3.2.1, $L_{X}(g) \in \mathscr{D}_{\mathfrak{l}}(X)$. Choose then an element $e \in \mathcal{E}(L, \alpha)$ such that $\hat{e}=L_{X}(g)$ (see 5.1) and define $\rho_{e}: \mathbf{H}(L, \alpha ; \mathfrak{h}) \rightarrow \mathbf{H}(L, \alpha ; \mathfrak{h})$ by

$$
\rho_{e}(s)=\left(\beta_{g} \circ p_{D}\right)^{1 / 2}\left(e \circ s \circ L_{X}(g)^{-1}\right), \quad s \in \mathcal{H}(L, \alpha ; \mathfrak{h}) .
$$

We shall prove that $\rho_{e}(s)$ satisfies (5.3.1) and

$$
\int_{G / D}\left\|\rho_{e}(s)_{D}(u)\right\|^{2} d \mu(u)=\int_{G / D}\left\|s_{D}(u)\right\|^{2} d \mu(u),
$$

which means that $\rho_{e} \in \mathbf{U}(\mathbf{H}(L, \alpha ; \mathfrak{h}))$. Indeed, keeping in mind Lemma 4.3.1, we have

$$
\begin{aligned}
\nabla_{q_{*}\left(L(a)_{*} x\right) \rho_{e}(s)=} & q_{*}\left(L(a)_{*} x\right)\left(\beta_{g} \circ p_{D}\right)^{1 / 2}\left(e \circ s \circ L_{X}(g)^{-1}\right) \\
& +\left(\beta_{g} \circ p_{D}\right)^{1 / 2} \nabla_{q_{*}\left(L(a)_{*} x\right)}\left(e \circ s \circ L_{X}(g)^{-1}\right) \\
= & \left(q_{D}\right)_{*}\left(L(a)_{*} x\right)\left(\beta_{g}\right)^{1 / 2}\left(e \circ s \circ L_{X}(g)^{-1}\right) \\
& +\left(\beta_{g} \circ p_{D}\right)^{1 / 2}\left(e \circ \nabla_{q_{*}\left(L(g a)_{*} x\right)} s\right)=0,
\end{aligned}
$$

for any $x \in \mathfrak{h}$ and $a \in G$. This proves that $\rho_{e}(s)$ satisfies (5.3.1). On the other hand, (5.3.3) is a direct consequence of (4.3.1).

Now if $e^{\prime}$ is another element of $\mathscr{E}(L, \alpha)$ such that $\hat{e}^{\prime}=L_{X}(g)$, then, by (5.1.1), $e^{\prime}=t e$ with $t \in T$, whence $\rho_{e^{\prime}}=t \rho_{e}$. Thus $\rho_{e}$ determines a welldefined element in $\mathbf{P U}(\mathbf{H}(L, \alpha ; \mathfrak{h}))$ which will be denoted $\pi(L, \alpha ; \mathfrak{h})(g)$. One sees without any difficulty that $g \mapsto \pi(L, \alpha ; \mathfrak{h})(g)$ is a homomorphism of $G$ in $\operatorname{PU}(\mathbf{H}(L, \alpha ; \mathfrak{h}))$. Moreover, the following result, whose proof we defer until the next section, holds true.

5.3.1. Proposition. $\pi(L, \alpha ; \mathfrak{h})$ is a projective representation of $G$ on $\mathbf{H}(L, \alpha$; h).

Clearly, if $\left(L^{\prime}, \alpha^{\prime}\right)$ is another representative in the class $\ell$, then $\pi\left(L^{\prime}, \alpha^{\prime} ; \mathfrak{h}\right)$ 
is projectively equivalent to $\pi(L, \alpha ; \mathfrak{h})$. Owing to this fact we may denote the equivalence class of $\pi(L, \alpha ; \mathfrak{h})$ by $\pi_{\ell, \mathfrak{h}}$.

5.4. We shall state in the remainder of this section our main results. The group $G$ will be assumed in what follows not only connected and simply connected as before, but also solvable. The proofs of the results stated below will be given in $\$ 6$.

5.4.1. THEOREM. Let $\omega \in Z_{0}^{2}(\mathfrak{g})$.

(i) The set of positive, nil-admissible polarizations of $g$ at $\omega$ is nonvoid.

(ii) For any positive, nil-admissible polarization $\mathfrak{h}$ of $\mathfrak{g}$ at $\omega$ and any $\ell=$ $[(L, \alpha)] \in \mathscr{L}_{c}\left(X_{\omega}, \theta_{\omega}\right)$ the projective representation $\pi(L, \alpha ; \mathfrak{h})$ is irreducible.

(iii) If $\mathfrak{h}$ and $\mathfrak{h}^{\prime}$ are two positive, nil-admissible polarizations of $\mathfrak{g}$ at $\omega$ and $\ell \in \mathcal{L}_{c}\left(X_{\omega}, \theta_{\omega}\right)$, then $\pi_{\ell, \mathfrak{h}}=\pi_{\ell, \mathfrak{h}}$.

The choice of the polarization $\mathfrak{h}$ being thus immaterial, we may denote the class $\pi_{\mathfrak{l}, \mathfrak{h}} \in G^{\Pi}$ simply by $\pi_{\mathfrak{k}}$

We shall define now an action of $G$ on the set $\mathcal{L}_{G}=\cup_{\omega} \mathscr{L}_{c}\left(X_{\omega}, \theta_{\omega}\right)$, $\omega$ running over $Z_{0}^{2}(g)$, as follows. For $g \in G, \omega \in Z_{0}^{2}(g)$ and $l=[(L, \alpha)] \in$ $\mathcal{L}_{c}\left(X_{\omega}, \theta_{\omega}\right)$ let $g^{-1} \cdot \ell$ denote the equivalence class of the pull-back of $(L, \alpha)$ by the diffeomorphism $i\left(g^{-1}\right):\left(X_{g \cdot \omega}, \theta_{g \cdot \omega}\right) \rightarrow\left(X_{\omega}, \theta_{\omega}\right)$, where $g \cdot \omega$ stands for $\operatorname{Ad}^{*}(g) \omega$ (see 3.3). It is an easy matter to see that $(g, \ell) \mapsto g^{-1} \cdot \ell$ is a well-defined action of $G$ on $\mathcal{L}_{G}$.

5.4.2. TheOREM. Let $\ell, \ell^{\prime} \in \mathfrak{L}_{G}$. Then $\pi_{\ell}=\pi_{\ell}$ if and only if there exists $g \in G$ such that $\ell^{\prime}=g^{-1} \cdot \ell$.

In other terms, the map $\ell \mapsto \pi_{\ell}$ from $\mathscr{L}_{G}$ to the projective dual $G^{\Pi}$ of $G$ factorizes through an injective map from $\mathfrak{L}_{G} / G$ into $G^{\Pi}$.

To state our next result we need two more definitions.

We shall say that $\omega \in Z^{2}(\mathrm{~g})$ is a type I cocycle if the simply connected Lie group $M_{\omega}$ with the Lie algebra $\mathfrak{m}_{\omega}$ (see 1.4) is of type $I$.

5.4.3. LEMMA: Any type I cocycle $\omega \in Z^{2}(g)$ is integral.

Obviously, if $\omega$ is a type I cocycle then $\operatorname{Ad}^{*}(g) \omega$ is also of type $I$, for any $g \in G$. Thus $\mathfrak{L}_{G}^{\mathrm{I}}=\cup_{\omega} \mathcal{L}_{c}\left(X_{\omega}, \theta_{\omega}\right), \omega$ running over the set $Z_{\mathrm{I}}^{2}(g)$ of all type $\mathrm{I}$ cocycles in $Z^{2}(\mathrm{~g})$, is a $G$-stable subset of $\mathscr{L}_{G}$.

Finally, the set of all equivalence classes of irreducible projective representations $\pi$ of $G$ such that the associated group $M_{\pi}$ (see 2.2 ) is of type I will be denoted by $G_{I}^{\Pi}$.

5.4.4. THEOREM. (i) $\ell \in \mathcal{L}_{G}^{\mathrm{I}}$ if and only if $\pi_{\ell} \in G_{\mathrm{I}}^{\Pi}$.

(ii) The map $\ell_{\mapsto} \mapsto \pi_{\ell}$ induces a bijection between $\mathfrak{L}_{G}^{\mathrm{I}} / G$ and $G_{\mathrm{I}}^{\Pi}$.

In the special case of exponential Lie groups the above result provides a complete parametrization of the projective dual. 
5.4.5. Corollary. Assume $G$ exponential. Then each $\omega \in Z^{2}(\mathrm{~g})$ is of type $\mathrm{I}$, and the set $\mathcal{L}_{c}\left(X_{\omega}, \theta_{\omega}\right)$ reduces to a single element $\ell(\omega)$. Furthermore, the map $\omega \mapsto \pi_{\mathfrak{\ell}(\omega)}$ from $Z^{2}(\mathrm{~g})$ to $G^{\Pi}$ factorizes through a bijection of $Z^{2}(\mathrm{~g}) / G$ onto $G^{\Pi}$.

\section{Proofs.}

6.1. Returning for the moment to the general case when $G$ is not necessarily solvable, let $\omega \in Z^{2}(\mathfrak{g})$, $\mathfrak{h}$ be a closed, positive polarization of $\mathfrak{g}$ at $\omega$, and $X=X_{\omega}, q=q_{\omega}, \theta=\theta_{\omega}, F=F_{\omega}, \mathfrak{m}=\mathfrak{m}_{\omega}, M=M_{\omega}, p=p_{\omega}, \nu=\nu_{\omega}, D, E$, $\tilde{\mathfrak{h}}, \tilde{D}, \tilde{E}$ be the associated data (see $3.2,4.1,4.2)$. Set $Y=M / M(\nu)$, where $M(\nu)=\left\{m \in M ; \operatorname{Ad}^{*}(m) \nu=\nu\right\}$ and let $\tilde{q}: M \rightarrow Y$ be the canonical projection. Then $d \nu$ induces a $M$-invariant 2 -form $\sigma=\sigma_{\omega}$ on $Y$ such that $(Y, \sigma)$ is a symplectic $M$-homogeneous space (see [3]). In view of (4.2.1) and (3.1.3), $M(\nu)=p^{-1}(G(\omega))$, so that $p: M \rightarrow G$ induces an isomorphism $\tilde{p}$ : $(Y, \sigma) \rightarrow(X, \theta)$ of symplectic spaces which has the additional property

$$
\tilde{p}\left(L_{Y}(m) u\right)=L_{X}(p(m)) \tilde{p}(u), \quad m \in M, u \in Y .
$$

Assume now $\omega$ integral and let $\ell=[(L, \alpha)] \in \mathfrak{L}_{c}(X, \theta)$. Of course, $(L, \alpha)$ can be viewed also as a line bundle with connection and invariant Hermitian structure over $Y$, the projection being this time $\tilde{p}^{-1} \circ{ }^{\circ} r_{L}$; when regarded as such, its equivalence class in $\mathscr{L}_{c}(Y, \sigma)$ will be denoted $\tilde{\ell}$. According to Theorem 5.7.1 in [3], there is a unique character $\eta=\eta_{p}: M(\nu) \rightarrow \mathrm{T}$ whose differential is $2 \pi i v \mid \mathfrak{m}(\nu)(\mathfrak{m}(\nu)$ being Lie $M(\nu))$, such that $\tilde{\ell}=[(\tilde{L}, \tilde{\alpha})]$, where $\tilde{L}=M \times_{\eta} \mathbf{C}$ is the line bundle over $Y$ associated to the principal bundle $\tilde{q}$ : $M \rightarrow Y$ with structure group $M(\nu)$ and to the representation $\eta: M(\nu) \rightarrow \mathbf{T}=$ $\mathbf{U}(\mathbf{C})$, and $\tilde{\alpha}$ is the "push-down" of the 1 -form $\left(\delta_{\nu},(1 / 2 \pi i)(d z / z)\right)$ on $M \times \mathbf{C}$, $\delta_{\nu}$ being the left invariant 1 -form corresponding to $\nu \in \mathrm{m}^{*}$. In more detail, $M \times{ }_{\eta} \mathbf{C}$ is obtained as the orbit space $(M \times C) / M(\nu)$, where the action of $M(\nu)$ on $M \times C$ is given by: $n \cdot(m, z)=\left(m n^{-1}, \eta(n) \cdot z\right), n \in M(\nu),(m, z)$ $\in M \times \mathbf{C}$. The projection $p r_{\tilde{L}}: \tilde{L} \rightarrow Y$ is the map $[m, z] \mapsto \tilde{q}(m)$, where $[m, z]$ stands for the orbit of $M(v)$ through $(m, z)$, while the linear and the Hermitian structure on $\tilde{L}$ are those determined by the usual ones on $\mathbf{C}$.

As it is known, $\eta$ extends to a unique character $\chi=\chi_{\ell}$ of $\tilde{D}$ whose differential is $2 \pi i \nu \mid \tilde{\delta}$. Now let $\rho(\chi, \tilde{\mathfrak{h}})$ be the holomorphically induced unitary representation of $M$ corresponding to the polarization $\tilde{\mathfrak{h}}$ and to the character $\chi$ (see [1]).

6.1.1. Lemma. The unitary representation $\rho(\chi, \tilde{\mathfrak{h}})$ of $M$ is projectable and the corresponding projective representation $\hat{\rho}(\chi, \tilde{\mathfrak{h}})$ of $G$ is projectively equivalent to $\pi(L, \alpha ; \mathfrak{h})$.

Proof. Recall first that the Hilbert space $\mathbf{H}(\chi, \tilde{\mathfrak{h}})$ on which $\rho(\chi, \tilde{\mathfrak{h}})$ acts comes from the vector space $\mathcal{H}(\chi, \tilde{\mathfrak{h}})$ of all $C^{\infty}$-functions $f: M \rightarrow C$ satisfying:

(i) $f(m d)=\chi(d)^{-1} f(m)$ for any $m \in M$ and $d \in \tilde{D}$; 
(ii) $l(x) f+2 \pi i\langle\nu, x\rangle f=0$ for any $x \in \tilde{\mathfrak{h}}$;

(iii) $\int_{M / \tilde{D}}|f(m)|^{2} d \tilde{\mu}(m \tilde{D})<\infty$,

where $\tilde{\mu}$ is the volume element on $M / \tilde{D}$ corresponding to $\mu$ via the isomorphism

$$
M / \tilde{D} \stackrel{\sim}{\sim} G / D
$$

induced by $p: M \rightarrow G$ (cf. (4.2.5)). The action of $\rho(\chi, \tilde{\mathfrak{h}})$ on $\mathbf{H}(\chi, \tilde{\mathfrak{h}})$ is expressed by the formula

$$
(\rho(\chi, \mathfrak{h})(m) f)\left(m^{\prime}\right)=\left(\beta_{p(m)}\left(q_{D}\left(p\left(m^{\prime}\right)\right)\right)\right)^{1 / 2} f\left(m^{-1} m^{\prime}\right), \quad m, m^{\prime} \in M .
$$

There is no problem in verifying that $\rho(\chi, \tilde{\mathfrak{h}})$ is projectable so that we do not insist on this point. In order to prove the second assertion, let us note first that $(\tilde{L}, \tilde{\alpha})$, when regarded as a line bundle with connection and Hermitian structure over $X$ (the corresponding projection being $\tilde{p} \circ p r_{\tilde{L}}$ ), is obviously equivalent to $(L, \alpha)$. Thus, for our purposes, there is no loss of generality in assuming $(L, \alpha)=(\tilde{L}, \tilde{\alpha})$ as line bundles over $X$. Now for each $f \in \mathcal{H}(\chi, \tilde{\mathfrak{h}})$, let $s_{f}: X \rightarrow L$ be the section

$$
s_{f}(q(p(m)))=[m, f(m)], \quad m \in M .
$$

By a routine computation one checks that $s_{f} \in \mathcal{H}(L, \alpha ; \mathfrak{h})$ and that the assignment $f \mapsto s_{f}$ gives rise to a unitary isomorphism $U: \mathbf{H}(\chi, \tilde{\mathfrak{h}}) \rightarrow \mathbf{H}(L, \alpha ; \mathfrak{h})$. Let $g \in G$ and choose $m \in M$ such that $p(m)=g ; m$ defines an element $e_{m} \in \mathcal{E}(L, \alpha)$ by $e_{m}\left(\left[m^{\prime}, z^{\prime}\right]\right)=\left[m^{\prime}, z^{\prime}\right]$, and clearly

$$
s_{\rho(\chi, \tilde{\mathfrak{h}})(g) f}=\left(\beta_{g} \circ p_{D}\right)^{1 / 2}\left(e_{m} \circ s_{f} \circ L_{X}(g)^{-1}\right), \quad f \in \mathcal{H}(\chi, \tilde{\mathfrak{h}}),
$$

which means that

$$
\hat{U} \circ \hat{\rho}(\chi, \tilde{\mathfrak{h}})(g)=\pi(L, \alpha ; \mathfrak{h})(g) \circ \hat{U} .
$$

This proves both the fact that $\pi(L, \alpha ; \mathfrak{h})$ is indeed a projective representation, as asserted in Proposition 5.3.1, and the claim of the present lemma.

6.2. In the rest of this section $G$ will be assumed solvable. All the notation remains as above.

6.2.1. Proof of TheORem 5.4.1. It suffices to combine (4.2.3), Lemma 6.1.1 and Theorems II.3.2, III.4.1, IV.5.7 from [1].

6.2.2. Proof OF TheOrem 5.4.2. Let us begin by proving that if $\ell \in$ $\mathcal{L}_{c}(X, \theta)$ (with $\left.X=X_{\omega}, \theta=\theta_{\omega}\right)$ and $\ell^{\prime} \in \mathcal{L}_{c}\left(X^{\prime}, \theta^{\prime}\right)\left(\right.$ with $X^{\prime}=X_{\omega^{\prime}}, \theta^{\prime}=\theta_{\omega^{\prime}}$ ) are related by the equality $\ell^{\prime}=g^{-1} \cdot \ell$, then $\pi_{\ell}=\pi_{\mathfrak{q}}$

Note first that $\omega^{\prime}=\operatorname{Ad}^{*}(g) \omega$, therefore, after choosing a positive, niladmissible polarization $\mathfrak{h}$ of $\mathfrak{g}$ at $\omega, \mathfrak{h}^{\prime}=\operatorname{Ad}(g) \mathfrak{h}$ will be a polarization of the same type at $\omega^{\prime}$. Further, pick $(L, \alpha) \in \ell$; then $\left(L^{\prime}, \alpha^{\prime}\right)=i\left(g^{-1}\right)^{*}(L, \alpha) \in \ell^{\prime}$. All the data concerning $\omega^{\prime}, \mathfrak{h}^{\prime}, \ell^{\prime}$ will be denoted by the same symbol as those 
attached to $\omega, \mathfrak{h}, \ell$, but affected by an accent. This convention will be valid all over this section.

Put $\lambda=F(g)$; then, by (3.1.6), $\omega^{\prime}-\omega=d \lambda$. Let $\varphi_{\lambda}: \mathfrak{m} \rightarrow \mathfrak{m}^{\prime}$ and $\Phi_{\lambda}$ : $M \rightarrow M^{\prime}$ be the corresponding Lie homomorphisms, as in 1.4. Now choose $m \in M$ such that $p(m)=g$ and let $\Lambda=\varphi_{\lambda}^{*} \nu^{\prime}-\operatorname{Ad}_{M}^{*}(m) \nu$. Then

$$
d\left(\varphi_{\lambda}^{*} \nu^{\prime}\right)=\varphi_{\lambda}^{*} d \nu^{\prime}=\varphi_{\lambda}^{*}\left(\psi^{\prime *} \omega^{\prime}\right)=\psi^{*} \omega^{\prime},
$$

while

$$
d\left(\operatorname{Ad}_{M}^{*}(m) \nu\right)=\operatorname{Ad}_{M}^{*}(m) d \nu=\operatorname{Ad}_{M}^{*}(m) \psi^{*} \omega=\psi^{*}\left(\operatorname{Ad}_{G}^{*}(g) \omega\right)=\psi^{*} \omega^{\prime},
$$

so that $d \Lambda=0$, which means that $\Lambda$ is a character of $m$. Hence $\tilde{\mathfrak{h}}_{m}=\operatorname{Ad}(m) \tilde{\mathfrak{h}}$ is a polarization for $\varphi_{\lambda}{ }^{*} \nu^{\prime}=\operatorname{Ad}_{M}^{*}(m) \nu+\Lambda$. Since $M$ is connected and simply connected, $\Lambda$ gives rise to a character $\chi_{\Lambda}$ of $M$ whose differential is $2 \pi i \Lambda$.

Let now $\eta$ and $\chi$ be the characters of $M(\nu)$ and $\tilde{D}$ respectively, associated to $\ell$, as in 6.1. Since $\nu^{\prime}=\left(\operatorname{Ad}_{M}^{*}(m) \nu+\Lambda\right) \circ \varphi_{\lambda}^{-1}$, whence $M^{\prime}\left(\nu^{\prime}\right)=$ $\Phi_{\lambda}(I(m) M(\nu))$, we can define a character $\eta^{\prime}$ of $M^{\prime}\left(\nu^{\prime}\right)$ by putting $\eta^{\prime}=\left(\chi_{\Lambda}\right.$. $\left.\left(\chi \circ I\left(m^{-1}\right)\right)\right) \circ \Phi_{\lambda}^{-1} \mid M^{\prime}\left(\nu^{\prime}\right)$. Consider now the diagram

$$
\begin{array}{ccc}
M^{\prime} \times \mathbf{C} & \rightarrow & M \times \mathbf{C} \\
\downarrow^{\eta^{\prime}} & & \downarrow^{\eta} \\
Y^{\prime}=M^{\prime} / M^{\prime}\left(\nu^{\prime}\right) & \rightarrow & M / M(\nu)=Y \\
\tilde{p}^{\prime} \downarrow & & \downarrow \tilde{p} \\
X^{\prime}=G / G\left(\omega^{\prime}\right) & \stackrel{i\left(g^{-1}\right)}{\rightarrow} & G / G(\omega)=X
\end{array}
$$

where the top horizontal arrow is given by $\left[m^{\prime}, z\right] \mapsto\left[I\left(m^{-1}\right) \circ \Phi_{\lambda}^{-1}\left(m^{\prime}\right)\right.$, $\chi_{\Lambda}\left(m^{\prime}\right) z$, the middle horizontal arrow is induced by $I\left(m^{-1}\right) \circ \Phi_{\lambda}^{-1}: M^{\prime} \rightarrow M$ and the top vertical arrows are determined by projections onto the first factors followed by projections onto quotient spaces. The fact that this diagram commutes, which can be easily verified, together with the discussion about line bundles in 6.1, imply that, by composing the left vertical arrows, one obtains a line bundle with connection over $X^{\prime}$ which is equivalent to $\left(L^{\prime}, \alpha^{\prime}\right)$. This means that $\eta^{\prime}$ is precisely the character associated to $\ell^{\prime}$.

Denote for simplicity $\tilde{D}_{m}=I(m)(\tilde{D})$ and $\chi_{m}=\chi \circ I\left(m^{-1}\right) \mid \tilde{D}_{m}$. Since $\tilde{D}^{\prime}$ $=\Phi_{\lambda}\left(\tilde{D}_{m}\right)$, one can see that $\chi^{\prime}$, the extension of $\eta^{\prime}$ to $\tilde{D}^{\prime}$, is given by the formula

$$
\chi^{\prime}=\left(\left(\chi_{\Lambda} \mid \tilde{D}_{m}\right) \chi_{m}\right) \circ\left(\Phi_{\lambda}^{-1} \mid \tilde{D}^{\prime}\right) .
$$

Furthermore, $\rho\left(\chi^{\prime}, \tilde{\mathfrak{h}}^{\prime}\right)=\rho\left(\left(\left(\chi_{\Lambda} \mid \tilde{D}_{m}\right) \chi_{m}\right) \circ\left(\Phi_{\lambda}^{-1} \mid \tilde{D}^{\prime}\right), \tilde{\mathfrak{h}}^{\prime}\right)$ is, by [1, Proposition I.5.13], unitarily equivalent to $\rho\left(\left(\chi_{\Lambda} \mid \tilde{D}_{m}\right) \chi_{m}, \tilde{\mathfrak{h}}_{m}\right) \circ \Phi_{\lambda}^{-1}$; consequently $\rho\left(\chi^{\prime}, \tilde{\mathfrak{h}}^{\prime}\right) \circ \Phi_{\lambda}$ is unitarily equivalent to $\rho\left(\left(\chi_{\Lambda} \mid \tilde{D}_{m}\right) \chi_{m}, \tilde{\mathfrak{h}}_{m}\right)$. At this moment we observe that $\rho\left(\left(\chi_{\Lambda} \mid \tilde{D}_{m}\right) \chi_{m}, \tilde{\mathfrak{h}}_{m}\right)$ is unitarily equivalent to $\rho\left(\chi_{m}, \tilde{\mathfrak{h}}_{m}\right) \otimes \chi_{\Lambda}$, through the unitary isomorphism of $\mathbf{H}\left(\left(\chi_{\Lambda} \mid \tilde{D}_{m}\right) \chi_{m}, \tilde{\mathfrak{h}}_{m}\right)$ onto $\mathbf{H}\left(\chi_{m}, \tilde{\mathfrak{h}}_{m}\right)$ given by $f \mapsto \chi_{\Lambda} \cdot f$. Using [1, (IV.2.2)] for instance, $\rho\left(\chi_{m}, \tilde{\mathfrak{h}}_{m}\right)$ is seen to be unitarily 
equivalent to $\rho(\chi, \tilde{\mathfrak{h}})$. Summing up these last remarks it follows that $\rho\left(\chi^{\prime}, \tilde{\mathfrak{h}}^{\prime}\right) \circ \Phi_{\lambda}$ is unitarily equivalent to $\rho(\chi, \tilde{\mathfrak{h}}) \otimes \chi_{\Lambda}$. To conclude this part of the proof, that is to get $\pi_{\ell, \mathfrak{h}}=\pi_{\ell, \mathfrak{h}^{\prime}}$, it is enough to invoke Lemma 6.1.1.

Assume now, conversely, that $\pi_{\ell}=\pi_{\ell}$, that is $\pi(L, \alpha ; \mathfrak{h})$ and $\pi\left(L^{\prime}, \alpha^{\prime} ; \mathfrak{h}^{\prime}\right)$ are projectively equivalent, where $\omega, \omega^{\prime} \in Z_{0}^{2}(\mathfrak{g}), \mathfrak{h}$ (resp. $\left.\mathfrak{h}^{\prime}\right)$ is a positive, nil-admissible polarization of $g$ at $\omega$ (resp. $\left.\omega^{\prime}\right)$ and $\ell=[(L, \alpha)] \in \mathcal{L}_{c}(X, \theta)$, $\ell^{\prime}=\left[\left(L^{\prime}, \alpha^{\prime}\right)\right] \in \mathcal{L}_{c}\left(X^{\prime}, \theta^{\prime}\right)$. From Lemma 6.1.1 and 2.4 we deduce that there exists an equivalence of extensions $\Phi: M \rightarrow M^{\prime}$ with differential $\varphi: \mathfrak{m} \rightarrow \mathfrak{m}^{\prime}$ such that $\rho(\chi, \tilde{\mathfrak{h}})$ and $\rho\left(\chi^{\prime}, \tilde{\mathfrak{h}}^{\prime}\right) \circ \Phi$ are unitarily equivalent. But $\rho\left(\chi^{\prime}, \tilde{\mathfrak{h}}^{\prime}\right) \circ \Phi$ is unitarily equivalent to $\rho\left({ }^{\prime} \chi, \tilde{\mathfrak{h}}\right)$, where ' $\chi$ is the character of ${ }^{\prime} \tilde{D}=\Phi^{-1}\left(\tilde{D}^{\prime}\right)$ given by ' $\chi=\chi^{\prime} \circ\left(\left.\Phi\right|^{\prime} \tilde{D}\right)$ and ' $\tilde{\mathfrak{h}}=\varphi^{-1}\left(\tilde{\mathfrak{h}}^{\prime}\right)$, hence, by [1, Theorem IV.5.7], there exists $m \in M$ such that $\varphi^{*} \nu^{\prime}=\operatorname{Ad}_{M}^{*}(m) \nu$ and $\eta=$ $\eta^{\prime} \circ((\Phi \circ I(m)) \mid M(\nu))$. Now, looking at the construction of the line bundle with connection associated to a character, which has been recalled in 6.1 , it is easily seen that $\ell^{\prime}=p(m)^{-1} \cdot \ell$.

6.2.3. Proof of LemMa 5.4.3. The notation being as agreed, let us recall that $(X, \theta)$ and $(Y, \sigma)$ are isomorphic symplectic spaces. But then, in view of Theorem V.5.2 in [1], the cohomology class of $\sigma$ is integral, hence $\omega$ is integral.

6.2.4. Proof of Theorem 5.4.4. The first claim results from Lemma 6.1.1 and 2.2. To prove the second claim it suffices to show that an arbitrary irreducible projective representation $\pi$ of $G$ whose class belongs to $G_{I}^{\Pi}$ is equivalent to a projective representation of the form $\pi(L, \alpha ; \mathfrak{h})$. To this end, consider the "unitary lifting" $\rho=\check{\pi}: M=M_{\pi} \rightarrow \mathbf{P U}(\mathbf{H})$ (cf. 2.2). In view of [1, Theorem V.3.3], there exist $\nu \in \mathrm{m}^{*}$ and a character $\eta$ of $M(\nu)$ with differential $2 \pi i \nu \mid m(\nu)$ such that, if we choose a positive, strongly-admissible polarization $\tilde{\mathfrak{h}}$ of $\mathfrak{m}$ at $\nu$ and denote by $\tilde{D}$ its corresponding " $D$ "-group and by $\chi$ the corresponding extension of $\eta$ to $\tilde{D}$, then $\rho$ is unitarily equivalent to $\operatorname{ind}_{M}(\eta, \tilde{\mathfrak{h}})$, the holomorphically induced representation of $M$ associated to $\eta$ and $\tilde{\mathfrak{h}}$ (see [1]). Since $\mathbf{R}$ is central in $M$, the very definition $\operatorname{ind}_{M}(\eta, \tilde{\mathfrak{h}})$ ensures us that

$$
\operatorname{ind}_{M}(\eta, \tilde{\mathfrak{h}})(r)=\exp \{2 \pi i\langle\nu, r\rangle\} \mathrm{Id}, \quad r \in \mathbf{R} ;
$$

on the other hand, $\rho$ being projectable, we have

$$
\operatorname{ind}_{M}(\eta, \tilde{\mathfrak{h}})(r)=\exp \{2 \pi i r\} \mathrm{Id}, \quad r \in \mathbf{R} .
$$

Now, according to $\S 1$, we can assume $\mathfrak{m}=\mathfrak{m}_{\omega}$ for a suitable $\omega \in Z^{2}(g)$. Define $\lambda \in g^{*}$ by $\langle\lambda, x\rangle=\langle\nu,(0, x)\rangle$ and $\omega^{\prime}=\omega+d \lambda$. Further, let $\varphi_{\lambda}$ : $\mathfrak{m} \rightarrow \mathfrak{m}^{\prime}=\mathfrak{m}_{\omega^{\prime}}$ be the isomorphism associated to $\lambda$. Clearly, $\nu=\nu^{\prime} \circ \varphi_{\lambda}$, where $\nu^{\prime}=\nu_{\omega^{\prime}}$. Then

$$
\operatorname{ind}_{M}(\eta, \tilde{\mathfrak{h}}) \simeq \operatorname{ind}_{M^{\prime}}\left(\eta^{\prime}, \tilde{\mathfrak{h}}^{\prime}\right) \circ \Phi_{\lambda}
$$


where $M^{\prime}$ is the connected and simply connected Lie group with $\operatorname{Lie}\left(M^{\prime}\right)=$ $\mathfrak{m}^{\prime}, \Phi_{\lambda}: M \rightarrow M^{\prime}$ is the isomorphism whose differential is $\varphi_{\lambda}, \tilde{\mathfrak{h}}^{\prime}=\varphi_{\lambda}(\tilde{\mathfrak{h}})$, $\eta^{\prime}=\eta \circ\left(\Phi_{\lambda}^{-1} \mid M^{\prime}\left(\nu^{\prime}\right)\right)$. But $\operatorname{ind}_{M^{\prime}}\left(\eta^{\prime}, \tilde{\mathfrak{h}}^{\prime}\right)$ is just the representation of $M^{\prime}$ we have denoted $\rho\left(\chi^{\prime}, \tilde{\mathfrak{h}}^{\prime}\right)$, where $\chi^{\prime}=\chi \circ\left(\Phi_{\lambda}^{-1} \mid \tilde{D}^{\prime}\right)$ and $\mathfrak{h}^{\prime}=\psi_{\omega^{\prime}}\left(\tilde{\mathfrak{h}}^{\prime}\right)$. Letting $\left(L^{\prime}, \alpha^{\prime}\right)$ denote the line bundle with connection over $X_{\omega^{\prime}}$ associated to $\eta^{\prime}$, one sees that $\pi\left(L^{\prime}, \alpha^{\prime} ; \mathfrak{h}^{\prime}\right)$ is projectively equivalent to $\pi$.

6.2.5. Proof of Corollary 5.4.5. Since a central extension by $\mathbf{R}$ of an exponential group is again an exponential group and since such a group, when connected and simply connected, is of type $I$, it follows that $Z^{2}(g)=$ $Z_{\mathrm{I}}^{2}(\mathrm{~g})$ and $G^{\Pi}=G_{\mathrm{I}}^{\Pi}$. Let now $\omega \in Z^{2}(\mathrm{~g})$. Because $M_{\omega}\left(\nu_{\omega}\right)$ is connected and simply connected, $\varrho_{c}\left(X_{\omega}, \theta_{\omega}\right)$ consists of only one element. The rest of the proof is merely a simple consequence of Theorem 5.4.4.

\section{REFERENCES}

1. L. Auslander and B. Kostant, Polarization and unitary representations of solvable Lie groups, Invent. Math. 14 (4) (1971), 255-354.

2. B.-Y. Chu, Symplectic homogeneous spaces, Trans. Amer. Math. Soc. 197 (1974), 145-159.

3. B. Kostant, Quantization and unitary representations, Lecture Notes in Math., no. 170, Springer, New York, 1970, 87-208.

4. D. Montgomery and L. Zippin, Topological transformation groups, Interscience, New York, 1955.

Institute of Mathematics, Str. Academiei 14, 78015 Bucharest, Romania 